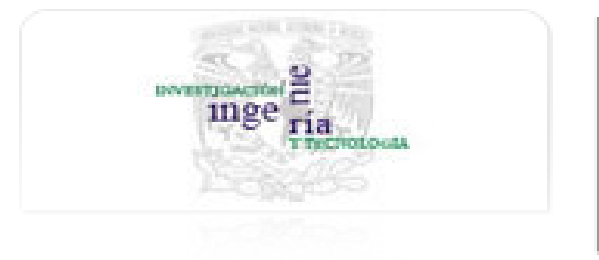

Ingeniería. Investigación y Tecnología ISSN: $1405-7743$

iit.revista@gmail.com

Universidad Nacional Autónoma de México

México

Mendoza Domínguez, A.; Graniel Peralta, M.

Estimación de emisiones a la atmósfera provenientes de quemadores elevados de instalaciones petroleras en la Sonda de Campeche

Ingeniería. Investigación y Tecnología, vol. VII, núm. 3, julio-septiembre, 2006, pp. 165-173

Universidad Nacional Autónoma de México

Distrito Federal, México

Disponible en: http://www.redalyc.org/articulo.oa?id=40470303

Cómo citar el artículo

Número completo

- Más información del artículo

- Página de la revista en redalyc.org

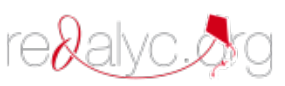

Sistema de Información Científica

Red de Revistas Científicas de América Latina, el Caribe, España y Portugal Proyecto académico sin fines de lucro, desarrollado bajo la iniciativa de acceso abierto 


\title{
Estimación de emisiones a la atmósfera provenientes de quemadores elevados de instalaciones petroleras en la Sonda de Campeche
}

\author{
A. Mendoza-Domínguez y M. Graniel-Peralta \\ Departamento de Ingeniería Q uímica y Centro de Calidad A mbiental, ITESM Campus M onterrey \\ E-mails: mendoza.alberto@ itesm.mx y mgranielp@pep.pemex.com
}

(Recibido: mayo del 2005; aceptado: noviembre de 2005)

\section{Resumen}

PEMEX Exploración y Producción (PEP), realiza venteos de gas asociado a través de quemadores elevados tipo antorcha en sus instalaciones de producción de la Sonda de Campeche, lo cual permite controlar las presiones en los separadores y líneas de conducción de dichas instalaciones. Este estudio se centra en cuantificar las emisiones atmosféricas provenientes de los quemadores pertenecientes a la Región Marina Noreste de PEP. Para ello, se elaboró un inventario de emisiones para seis diferentes episodios de estudio entre noviembre de 2000 y noviembre de 2001, a fin de diagnosticar el comportamiento de las emisiones a través de diferentes condiciones meteorológicas. El inventario presentado fue para $\mathrm{CO}, \mathrm{CO}_{2}, \mathrm{SO}_{2}$, óxidos de nitrógeno, partículas suspendidas totales e hidrocarburos no quemados. El inventario se construyó empleando factores de emisión, en los cuales se factorizaron ineficiencias de combustión teóricas, debidas a las condiciones meteorológicas imperantes en la zona. Los resultados obtenidos, muestran emisiones considerables de $\mathrm{H}_{2} \mathrm{~S}$ y compuestos orgánicos no quemados por ineficiencias de combustión en los quemadores, estimadas entre $22 \%$ y $44 \%$.

Descriptores: Contaminación atmosférica, inventario de emisiones, combustión, factores de emisión, plataformas marinas.

\footnotetext{
Abstract

PEM EX Exploration and Production (PEP) per forms vent ing of as so ci ated gas through high torch-type burn ers in its pro duc tion fa cil i ties in the south ern most part of the $G$ ulf of $M$ exico (Sonda deCampeche), al lowing con trol of op er a tion pres su res of sep a ra tors and pipelines in such fa cil i ties. This study fo cuses in es ti mat ing theamount of at mo spheric emis sions originated from burn ers in PEP's $N$ orth east $M$ a rine Region. Thus, an emis sion in ven tory for six differ e $n t$ study ep i sodes between $\mathrm{N}$ o vem ber 2000 and $\mathrm{N}$ o vem ber 2001 was elab o rated, with theob jective of di agnosing theemissions' behaviorsthrough out differ en tmeteo rologi cal con di tions. The in ven tory was gen er ated for $\mathrm{CO}, \mathrm{CO}_{2}, \mathrm{SO}_{2}$, ni tro gen ox ides, to tal sus pended par ti cles and un burned hy drocar bons. Thein ven tory was as sem bled using emis sion factors that account for the o ret i cal com bustion in effi cien cies dueto meteo rologi cal con ditions. Theresults show con siderable amounts of $\mathrm{H}_{2} \mathrm{~S}$ and un burned or ganic com pounds dueto com bus tion in ef fi cien cies that ranged be tween $22 \%$ and $44 \%$.
} 
Estimación de emisiones a la atmósfera provenientes de quemadores elevados ...

\section{Introducción}

La industria petrolera es uno de los ramos industriales más importantes para nuestro país. El organismo responsable de la exploración y explotación de los yacimientos de hidrocarburos del país es PEMEX Exploración y Producción, siendo las Regiones Marinas Noreste (RMNE) y Suroeste (RMSO) las encargadas de realizar las actividades de extracción y transporte de petróleo crudo y gas natural en el Sur del Golfo de México, específicamente en la Sonda de Campeche (Figura 1). De esta zona se extrae más del $80 \%$ de la producción nacional de petróleo crudo, del cual, el $85 \%$ le corresponde a la RMNE, el $15 \%$ a la RMSO y el $33 \%$ a la producción del gas natural, perteneciéndole el $60 \%$ a la RMNE y el $40 \%$ a la RMSO (PEMEX, 2004). De los activos que conforman la RMNE, Cantarell aporta más del $85 \%$ de la producción de petróleo crudo de dicha Región, y más del $70 \%$ del gas natural (PEMEX, 2004). El gas natural que se extrae en esta zona viene asociado al petróleo crudo. La mezcla de petróleo y gas extraída es separada en las instalaciones de producción, para posteriormente enviar el petróleo crudo a las Terminales Marítimas, Cayo Arcas y Dos Bocas. Por su parte, el gas natural asociado, conocido también como gas amargo por contener sulfuro de hidrógeno $\left(\mathrm{H}_{2} \mathrm{~S}\right)$ y bióxido de carbono $\left(\mathrm{CO}_{2}\right)$, es comprimido y enviado al Centro de Proceso y Transporte de Gas en la Península de Atasta (CPTGA), en Campeche.
Las instalaciones de producción cuentan con sistemas de desfogue a la atmósfera de sus corrientes gaseosas para evitar sobrepresionamientos en el sistema de compresión, en caso de alguna falla operativa o rechazo de gas en las instalaciones procesadoras. Sin embargo, debido al contenido de hidrocarburos explosivos y $\mathrm{H}_{2} \mathrm{~S}$ de la corriente gaseosa, ésta no se envía directamente a la atmósfera, dado que esto constituiría un riesgo para el personal que labora en las instalaciones. Cabe mencionar que el $\mathrm{H}_{2} \mathrm{~S}$ a concentraciones mayores de 250 partes por millón en volumen (ppmv) causa edema pulmonar y superiores a 1,000 ppmv puede ser mortal por causar parálisis respiratoria (US EPA, 1990). Para evitar riesgos, el gas amargo rechazado es enviado a quemadores elevados tipo "antorcha". Como resultado de la combustión del gas amargo, además de $\mathrm{CO}_{2}$ y agua, se emite bióxido de azufre $\left(\mathrm{SO}_{2}\right)$, óxidos de nitrógeno $\left(\mathrm{NO}_{\mathrm{x}}\right)$, partículas suspendidas (PS), monóxido de carbono (CO), compuestos orgánicos no quemados y $\mathrm{H}_{2} \mathrm{~S}$ no oxidado. Estimados recientes (Villasenor et al., 2003), indican que cerca del $82 \%$ del total de contaminantes emitidos a la atmósfera en las regiones marinas proviene de estas operaciones de quemado de gas.

En la actualidad, existe información limitada acerca de las emisiones de contaminantes atmosféricos provenientes de los activos de la RMNE, su destino y efectos que ocasionan al medio. Algunos autores han estudiado los efectos

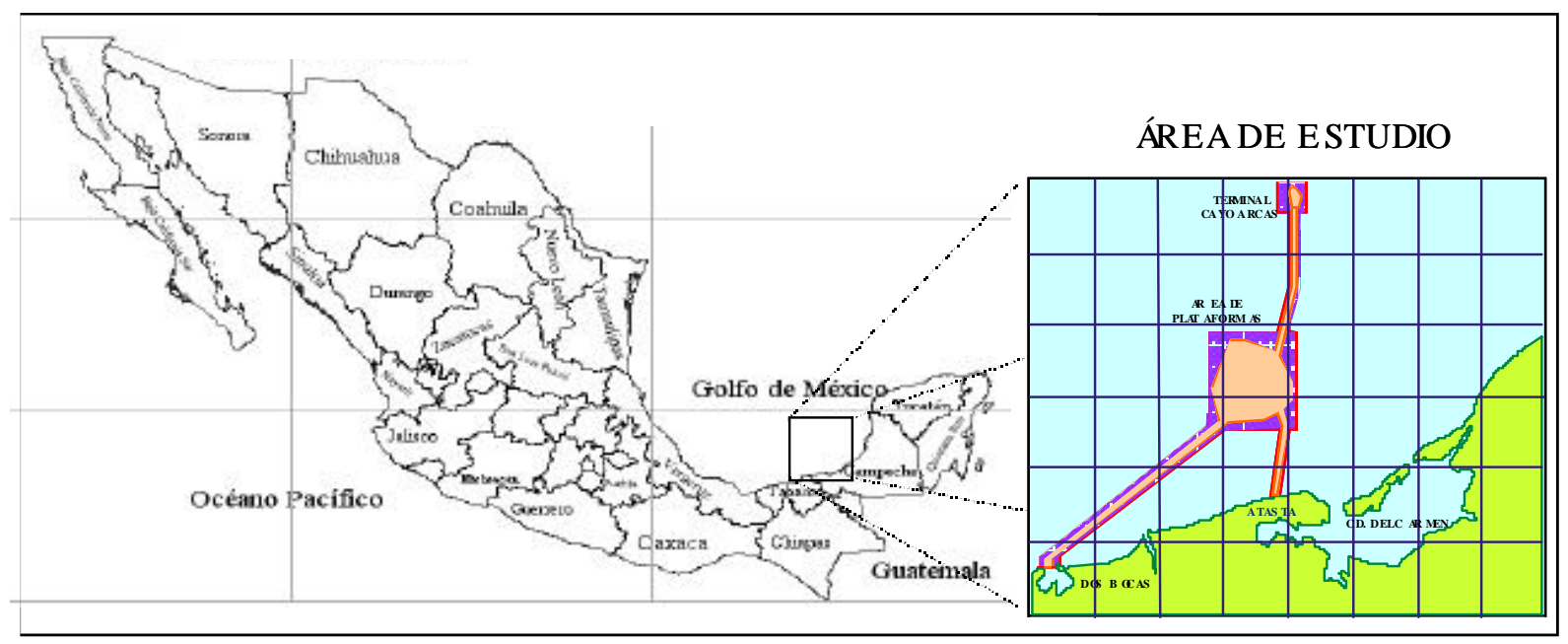

Figura 1. Ubicación de la zona de explotación petrolera en el Sur del Golfo de México 
de la lluvia ácida en el Golfo de México (Parungo et al., 1990; Bravo et al., 2000 y 2004), pero sin relacionar directamente las fuentes de emisión con los efectos ambientales. Gamboa-Rodríguez et al. (2004) realizaron un estudio para relacionar niveles de hidrocarburos aromáticos policíclicos en el aire ambiente con actividades de la industria petrolera, pero en zonas tierra adentro del Estado de Tabasco. Recientemente, Villaseñor et al. (2003), reportaron un inventario de emisiones integral de las operaciones de exploración y producción en la Sonda de Campeche. Sin embargo, el inventario reportado no considera el efecto de las ineficiencias de combustión en las emisiones de los quemadores elevados, debidas a condiciones meteorológicas locales. Así, en el presente artículo se presentan estimaciones de las emisiones provenientes de los quemadores elevados tipo antorcha de gas o perados por PEMEX en la RMNE derivadas de la aplicación de una técnica para calcular la eficiencia de los quemadores con base en las condiciones meteorológicas imperantes, y así corregir las estimaciones de las emisiones obtenidas puramente por factores de emisión. Esta información es relevante, ya que se puede emplear como base para estudiar posteriormente el efecto potencial de las emisiones a lo largo de la costa del Golfo de México, tanto en la salud de los habitantes en actividades como la agricultura y en los bienes materiales (por ejemplo, lluvia ácida), como en la calidad escénica (visibilidad) de la región.

\section{Metodología}

\section{Fuentes de información}

Debido a que la altura de los quemadores tipo antorcha típicamente sobrepasa los 18 metros y no se cuenta con tecnologías para la medición rutinaria de estas emisiones, generalmente se realiza una estimación de la emisión de los contaminantes empleando factores de emisión reportados en la literatura. En la figura 1 se muestra la zona en donde están ubicadas las instalaciones marinas de producción de la RMNE, las cuales fueron sujeto de este estudio. Se utilizó información correspondiente al período de noviembre del 2000 a noviembre del 2001 con el fin de observar las variaciones estacionales de las emisiones. En particular, se seleccionaron los períodos presentados en la tabla 1 como representativos de las diferentes estaciones del año. Un criterio fundamental para seleccionar estos períodos fue que no existieran huecos de información meteorológica necesaria para corregir las estimaciones de emisiones derivadas de la aplicación de los factores de emisión. En cuanto a los volúmenes de hidrocarburos enviados a quemadores, se utilizó la información de los Centros de proceso marinos Akal-C, Akal-J, Akal-N, Nohoch-A, Ku-A y Ku-H, así como la del CPTGA. Esta información fue proveída en promedios horarios. Asimismo, se utilizó información meteorológica horaria de cinco estaciones, ubicadas dentro del área de estudio para generar los campos meteorológicos requeridos en la estimación de emisiones. Las estaciones de donde provino la información fueron: Terminal Marítima Cayo Arcas, Terminal Marítima Dos Bocas, Plataforma de Telecomunicaciones Ixtoc-A, Plataforma de Telecomunicaciones Eco-1 y Plataforma de Rebombeo.

Tabla 1. Períodos de estudio seleccionados

\begin{tabular}{cc}
\hline Período & Fechas \\
\hline Otoño 1 & 01/Nov/2000-11/Nov/2000 \\
Otoño 2 & 05/Dic/2000-19/Dic/2000 \\
Invierno 1 & 21/Dic/2000-06/Ene/2001 \\
Invierno 2 & 11/Ene/2001-25/Ene/2001 \\
Primavera & 25/Mar/2001-05/Abr/2001 \\
Verano & $17 / \mathrm{Ago} / 2001-26 / \mathrm{Ago} / 2001$ \\
\hline
\end{tabular}

Generación del inventario de emisiones

Se estimaron las emisiones horarias de $\mathrm{SO}_{2}, \mathrm{H}_{2} \mathrm{~S}$, $\mathrm{NO}_{x}, \mathrm{CO}, \mathrm{CO}_{2}$, PS, y compuestos orgánicos totales (COT) provenientes de 16 quemadores elevados, así como un quemador de fosa. Para la formulación de los factores de emisión, se consideraron métodos desarrollados para operaciones de producción y de proceso de la industria petrolera (US EPA, 1995; ERG, 1999). La estimación de emisiones de $\mathrm{H}_{2} \mathrm{~S}, \mathrm{SO}_{2}$ y $\mathrm{CO}_{2}$, considera la razón de conversión como función de la eficiencia de la combustión. Por ejemplo, el cálculo de la emisión de $\mathrm{H}_{2} \mathrm{~S}$ (masa emitida por unidad de tiempo) se expresa como: 


$$
E_{\mathrm{H}_{2} \mathrm{~S}}=Q \ddot{\mathrm{Y}}\left(y_{\mathrm{H}_{2} \mathrm{~S}}\right) \ddot{\mathrm{Y}} \stackrel{\hat{\mathrm{E}}}{\hat{\mathrm{E}}} \frac{1}{\mathrm{C}}{ }_{\varnothing}{ }_{\varnothing} \ddot{\mathrm{Y}}\left(1-M_{\mathrm{SO}_{2}}\right) \ddot{\mathrm{Y}} P M_{\mathrm{H}_{2} S}
$$

(1)

donde $E_{\mathrm{H}_{2} \mathrm{~S}}$ es la emisión de $\mathrm{H}_{2} \mathrm{~S}, Q$ es el flujo volumétrico de gas quemado, $\mathrm{y}_{\mathrm{H}_{2} \mathrm{~S}}$ es la fracción molar de $\mathrm{H}_{2} \mathrm{~S}$ en el gas quemado, $\mathrm{C}$ es el volumen molar del gas ideal, $M_{\mathrm{O}_{2}}$ es la razón de conversión molar de $\mathrm{H}_{2} \mathrm{~S}_{a} \mathrm{SO}_{2}$ (basado en la estequiometría y suponiendo una conversión dependiente de la eficiencia de la combustión), y $P M_{\mathrm{H}, \mathrm{S}}$ es el peso molecular del $\mathrm{H}_{2} \mathrm{~S}$. Formas similares a la ecuación (1) fueron empleadas para estimar las emisiones de $\mathrm{SO}_{2}$ y $\mathrm{CO}_{2}$. Para las emisiones de $\mathrm{CO}$ y $\mathrm{NO}_{x}$ se consideran estimaciones de acuerdo al poder calorífico del gas quemado. Así, por ejemplo, para el compuesto $\mathrm{i}\left(\mathrm{i}=\mathrm{CO} \circ \mathrm{NO} \mathrm{C}_{x}\right.$ ) se tiene:

$$
E_{i}=Q \ddot{Y} F E_{i} \ddot{Y} P C_{G N}
$$

donde $\mathrm{E}_{i}$ es la emisión del compuesto i, $P C_{G N}$ es el poder calorífico del gas natural $(1,532.165$ $\mathrm{BTU} / \mathrm{ft}^{3}$, según datos proporcionados por PEMEX), y $F E_{\mathrm{i}}$ es el factor de emisión ( $F_{\mathrm{CO}}$ es igual a 0.37 $\mathrm{lb} / 10^{6}$ BTU, y $\mathrm{F}_{\mathrm{NO}}$ es igual a $\left.0.068 \mathrm{lb} / 10^{6} \mathrm{BTU}\right)$. Para el caso de las PS, se tomó un factor de emisión promedio de $6.77 \mathrm{lb} / 10^{6} \mathrm{ft}^{3}$ de gas quemado, el cual corresponde a quemadores con humeo medio. Finalmente, las emisiones de COT no quemados fueron estimadas mediante un balance de materia.

La información requerida para la estimación de la eficiencia de los quemadores incluyó, entre otros parámetros, dimensiones de cada uno de los quemadores, volumen de gas quemado, temperatura de la flama, temperatura ambiental y velocidad del viento. Esta información fue recopilada y usada para estimar las dimensiones de la flama, los efectos que causa el viento sobre la combustión, y posteriormente, la eficiencia de la combustión (Leahey et al., 2001). La formulación usada para la estimación de la eficiencia de combustión $(E)$ se basa en la relación de la cantidad estimada de energía emitida por la flama $\left(H_{f}\right)$ y la cantidad de energía contenida en el gas $(H)$ :

$$
E=\frac{100 H_{f}}{H}(\%)
$$

La estimación de la energía emitida por la flama $\left(H_{f}\right)$, se calcula como sigue:

$$
H_{f}=S+R^{\prime}
$$

en donde $S$ es el calor sensible ganado por el aire al pasar a través de la flama, y $R^{\prime}$ es el calor perdido por la flama, debido a la radiación. $R^{\prime}$ se calcula como:

$$
R^{\prime}=A_{f} s T_{f}^{4}
$$

donde $A_{f}$ es el área de la flama, $\sigma$ es la constante de Stefan-Boltzmann, y $T_{f}$ es la temperatura de la flama. El valor de $S$ (al igual que el de $A_{f}$ ) puede ser estimado, suponiendo que la flama se comporta como una flama difusiva, como:

$$
S=\frac{C p r_{f} V_{f}\left(T_{f}-T_{o}\right)}{t_{f}}
$$

donde $C p$ es el calor específico a presión constante del aire, $\rho_{\mathrm{f}}$ es la densidad del aire evaluada a $T_{f} V_{f}$ es el volumen ocupado por la flama, $T_{o}$ es la temperatura ambiente, $\mathrm{y} t_{f}$ es el tiempo necesario para que el aire pase a través de la flama $\mathbb{t}_{f}=$ $x_{f} / U$, donde $x_{f}$ es la longitud horizontal de la flama y $U$ es la velocidad del viento). Las dimensiones de la flama $\left(A_{f}, V_{f}\right.$ y $\left.x_{f}\right)$ se pueden calcular empleando el modelo desarrollado por Leahey et al. (1987), el cual no se presenta por brevedad.

Finalmente, la información meteorológica requerida (temperatura ambiente y velocidad de viento) para el cálculo de eficiencias de combustión, se obtuvo de campos generados por interpolación espacial. Esto fue necesario, ya que la posición de las estaciones meteorológicas no coincide con la posición de las plataformas de producción donde se encuentran los quemadores (Figura 2).

Así, los campos meteorológicos fueron obtenidos mediante un modelo de diagnóstico (Goodin et al., 1979) a partir de la información puntual de las estaciones meteorológicas de la zona. El modelo realiza una interpolación espacial con pesos que varían con el inverso del cuadrado de la distancia: 


$$
V_{\mathrm{int}}=\frac{\hat{\mathrm{A}} V_{m, i}\left(1 / d_{i}^{2}\right)}{\hat{\mathrm{A}}_{i}^{1 / d_{i}^{2}}}
$$

donde $V_{\text {int }}$ es el valor interpolado, $V_{m, i}$ es el valor medido en la estación i, y $d_{i}$ es la distancia de la estación al punto en donde se está generando el valor interpolado. El dominio seleccionado para realizar la interpolación espacial contiene 42 celdas en el eje horizontal y 56 celdas en el eje vertical, cada una de $4 \times 4 \mathrm{~km}^{2}$ (Figura 2). La esquina inferior izquierda del área establecida, se sitúa en la coordenada UTM 480,000 (m) en el eje horizontal y $2,016,000(\mathrm{~m})$ en el eje vertical, de la zona geográfica número 15. De esta forma, el modelo de diagnóstico genera valores interpolados en el centro de cada celda, los cuales son usados para el proceso de estimación de emisiones.

\section{Resultados}

Las variaciones propias del proceso de extracción de hidrocarburos del subsuelo, así como el origen del mismo, traen como consecuencia variaciones en la composición de los gases venteados. Con el fin de simplificar el análisis, se decidió tomar una composición promedio del gas asociado quemado (Tabla 2). La eficiencia global de combustión estimada de los quemadores durante los períodos de estudio fue de $22 \%$, lo que resultó en emisiones considerables de hidrocarburos no quemados y de $\mathrm{H}_{2} \mathrm{~S}$ no convertido a $\mathrm{SO}_{2}$. Los quemadores menos eficientes fueron los ubicados más lejos de la zona costera, debido principalmente a la velocidad de vientos encontrados en estos sitios. Los quemadores del CPTGA, registraron una eficiencia promedio del $44 \%$. Estos resultados son consistentes con estimaciones teóricas (Leahey et al., 2001) para quemadores operando con metano, debiéndose la alta ineficiencia a las flamas relativamente cortas que se generan con la consecuente dificultad de poder disipar eficientemente calor. Estos resultados fueron obtenidos suponiendo una temperatura de flama de $1200^{\circ} \mathrm{K}$ (Mendoza et al., 2004).

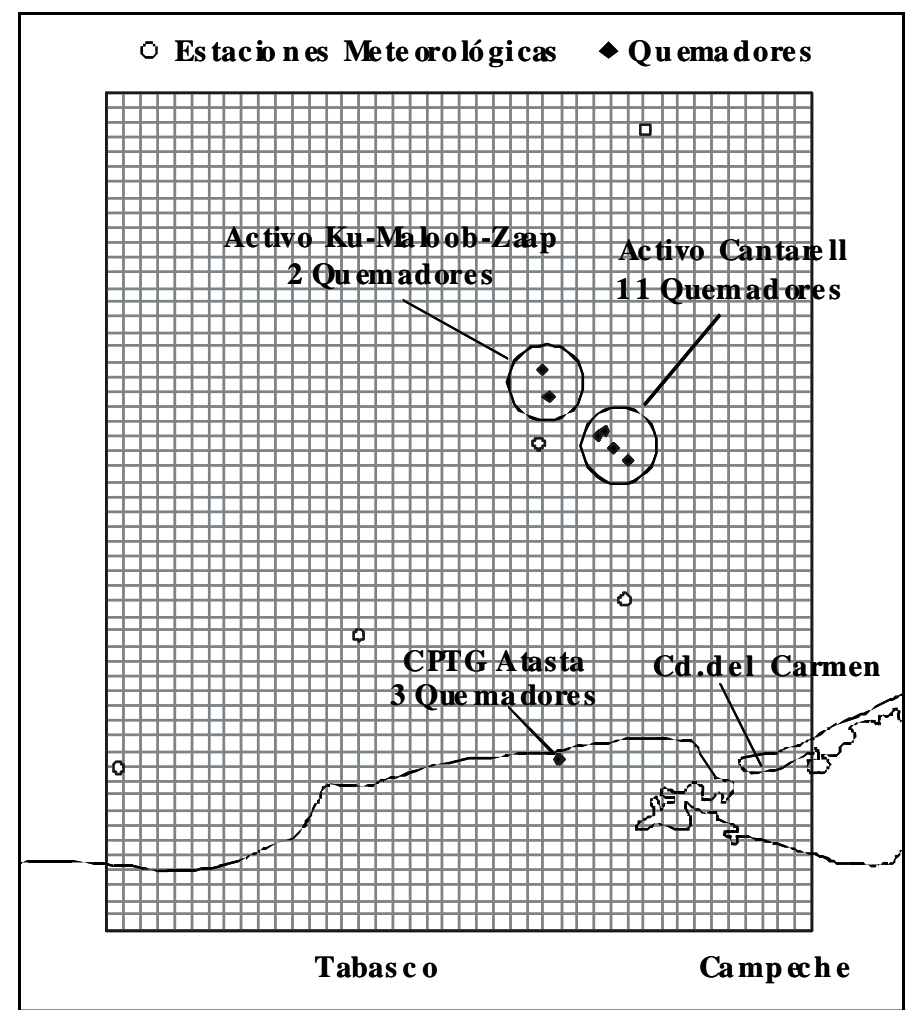

Figura 2. Detalle de la malla de interpolación y ubicación de los quemadores y estaciones meteorológicas en el área de estudio 
Estimación de emisiones a la atmósfera provenientes de quemadores elevados ...

Tabla 2. Composición promedio del gas asociado venteado

\begin{tabular}{cc}
\hline Compuesto & $\% \mathrm{~mol}$ \\
\hline $\mathrm{N}_{2}$ & 0.2868 \\
$\mathrm{CO}_{2}$ & 1.7925 \\
$\mathrm{H}_{2} \mathrm{~S}$ & 1.3971 \\
$\mathrm{H}_{2} \mathrm{O}$ & 2.6186 \\
$\mathrm{C}_{1}$ & 64.6776 \\
$\mathrm{C}_{2}$ & 12.7494 \\
$\mathrm{C}_{3}$ & 7.8023 \\
$\mathrm{i}-\mathrm{C}_{4}$ & 1.2815 \\
$\mathrm{n}-\mathrm{C}_{4}$ & 2.9755 \\
$\mathrm{i}-\mathrm{C}_{5}$ & 1.1232 \\
$\mathrm{n}-\mathrm{C}_{5}$ & 1.3903 \\
$\mathrm{C}_{6+}$ & 1.9052 \\
\hline
\end{tabular}

La tabla 3 presenta un resumen del volumen de gas enviado a quemadores y las emisiones estimadas producto de su combustión. En el perío do Invierno 2 , el volumen quemado fue considerablemente mayor que en los demás períodos, por consiguiente, la carga de contaminantes fue también superior en ese mismo período. En la tabla 4 se muestra con más detalle la carga total emitida por cada quemador. En ella, se puede apreciar que los quemadores con mayor cantidad de emisiones son: AK-C2 (15\%), NH-A1 (15\%) y KU-A1 (19\%) de los Centros de Procesos Akal-C, Nohoch-A y Ku-A, respectivamente. Los quemadores de la única instalación terrestre, CPTGA (CB-101, CB-102A y el CB-103B), sólo representan el $1.1 \%$ del total de las emisiones.

Tabla 3. Resumen de volumen quemado y emisiones generadas para cada período de estudio

\begin{tabular}{cccc}
\hline Período & $\begin{array}{c}\text { Volumen de gas quemado } \\
\left(\text { Millones } \mathrm{ft}^{3}\right)^{\mathrm{a}}\end{array}$ & $\begin{array}{c}\text { Emisiones totales } \\
\text { (Toneladas métricas) }\end{array}$ & $\begin{array}{c}\text { Promedio diario de emisiones por quemador } \\
\text { (Toneladas métricas) }\end{array}$ \\
\hline Otoño 1 & 3,087 & 122,211 & 690 \\
Otoño 2 & 1,626 & 65,042 & 271 \\
Invierno 1 & 3,692 & 146,865 & 541 \\
Invierno 2 & 6,205 & 240,409 & 1,000 \\
Primavera & 2,941 & 117,168 & 611 \\
Verano & 2,235 & 85,822 & 538 \\
\hline
\end{tabular}

a Volumen medido a condiciones PEMEX

Tabla 4. Emisiones totales (toneladas métricas) por quemador

\begin{tabular}{ccccccc}
\hline \multirow{2}{*}{ Quemador } & \multicolumn{7}{c}{ Período } \\
\cline { 2 - 7 } & Otoño 1 & Otoño 2 & Invierno 1 & Invierno 2 & Primavera & Verano \\
\hline AK-C1 & 1,198 & 487 & 1,389 & 2,302 & 19,541 & 6,520 \\
AK-C2 & 16,781 & 6,854 & 17,366 & 37,659 & 18,206 & 21,478 \\
AK-C3 & 2,900 & 1,179 & 3,506 & 6,130 & 16,758 & 5,976 \\
AK-C4 & 7,653 & 3,130 & 15,334 & 5,851 & 5,032 & 2,690 \\
AK-J1 & 2,583 & 1,060 & 1,632 & 7,827 & 1,281 & 2,012 \\
AK-J2 & 5,045 & 2,053 & 12,928 & 4,144 & 1,354 & 9,866 \\
AK-J3 & 8,371 & 3,423 & 18,161 & 12,556 & 7,003 & 1,906 \\
AK-J4 & 965 & 385 & 535 & 2,529 & 7,742 & 2,825 \\
AK-N1 & 24,928 & 10,118 & 8,002 & 1,322 & 5,175 & 4,820 \\
\hline & \multicolumn{7}{c}{} & continúa...
\end{tabular}


Tabla 4. Emisiones totales (toneladas métricas) por quemador (...continuación)

\begin{tabular}{ccccccc}
\hline \multirow{2}{*}{ Quemador } & \multicolumn{7}{c}{ Período } \\
\cline { 2 - 7 } & Otoño 1 & Otoño 2 & Invierno 1 & Invierno 2 & Primavera & Verano \\
\hline NH-A1 & 21,585 & 8,744 & 14,591 & 63,274 & 3,339 & 6,581 \\
NH-A2 & 960 & 260 & 15,530 & 53,883 & 3,387 & 3,022 \\
KU-A1 & 23,871 & 22,072 & 30,464 & 34,878 & 22,909 & 14,040 \\
KU-H1 & 4,345 & 4,005 & 5,525 & 6,226 & 4,194 & 2,494 \\
CB-101 & 191 & 279 & 383 & 325 & 237 & 326 \\
CB-102A & 470 & 583 & 956 & 1,006 & 568 & 752 \\
CB-103B & 365 & 409 & 565 & 497 & 441 & 514 \\
\hline
\end{tabular}

Tabla 5. Emisión por contaminante (toneladas métricas) para cada período de estudio

\begin{tabular}{cccccccc}
\hline \multirow{2}{*}{ Período } & \multicolumn{7}{c}{ Contaminante } \\
\cline { 2 - 8 } & $\mathrm{SO}_{2}$ & $\mathrm{H}_{2} \mathrm{~S}$ & $\mathrm{NO}_{\mathrm{X}}$ & $\mathrm{CO}$ & $\mathrm{PS}$ & $\mathrm{CO}_{2}$ & COT \\
\hline Otoño 1 & 653 & 1,410 & 146 & 794 & 9 & 49,837 & 69,361 \\
Otoño 2 & 357 & 736 & 77 & 418 & 5 & 27,263 & 36,185 \\
Invierno 1 & 795 & 1,679 & 174 & 950 & 11 & 60,651 & 82,604 \\
Invierno 2 & 1,209 & 2,889 & 293 & 1,597 & 19 & 92,236 & 142,166 \\
Primavera & 637 & 1,335 & 139 & 757 & 9 & 48,592 & 65,699 \\
Verano & 420 & 1,049 & 106 & 575 & 7 & 32,035 & 51,630 \\
\hline
\end{tabular}

Finalmente, en la tabla 5 se muestra un desglose de las emisiones de cada período por contaminante. Como es de esperarse, las emisiones de $\mathrm{CO}_{2}$ representan un porcentaje considerable del total emitido. Debido a las ineficiencias estimadas en la operación de los quemadores, las emisiones de $\mathrm{H}_{2} \mathrm{~S}$ son mayores a las de $\mathrm{SO}_{2}$, mientras que la emisión de hidrocarburos no quemados es apreciable.

\section{Discusión}

El inventario de emisiones generado, refleja variabilidades en las emisiones de gases enviados a la atmósfera en los diferentes períodos de análisis. Esta variabilidad es resultado de tres factores fundamentales. En primer lugar, por variaciones a través del año en los volúmenes de gas quemado, debidas primordialmente a cuestiones operativas (sobrepresionamientos); otra variable involucrada, es el origen del gas, ya que éste viene asociado al petróleo crudo que se extrae en el área marina, siendo diferente la fracción de gas en cada campo de extracción, y por ende, dependiendo de las tasas de extracción en cada campo será la cantidad de gas producido. En tercer lugar, se tiene que las variaciones meteorológicas impactan las eficiencias de los quemadores. En este último punto, resultados de otros autores (Leahey et al., 2001) indican una gran sensibilidad de la eficiencia de combustión a la velocidad del viento, más que cualquier otra variable, de tal manera que velocidades por encima de $4 \mathrm{~m} / \mathrm{s}$ llegan a reducir la eficiencia por debajo de un $40 \%$. Las velocidades de viento reportadas para el período de Noviembre de 2000 a 2001 (Figura 3) superan consistentemente los $4 \mathrm{~m} / \mathrm{s}$ en la zona marina (p. ej., estación Ico-1), mientras que en la zona costera las intensidades se ven disminuidas (estación Dos Bocas). Así, velocidades típicamente altas en las zonas marinas conllevan bajas eficiencias de combustión, tal como se presentó en este estudio. Estos resultados indican que un análisis posterior de los impactos de estas emisiones tiene que considerar la variabilidad encontrada al momento de generar los inventarios de emisiones en quemadores elevados. 
Estimación de emisiones a la atmósfera provenientes de quemadores elevados ...

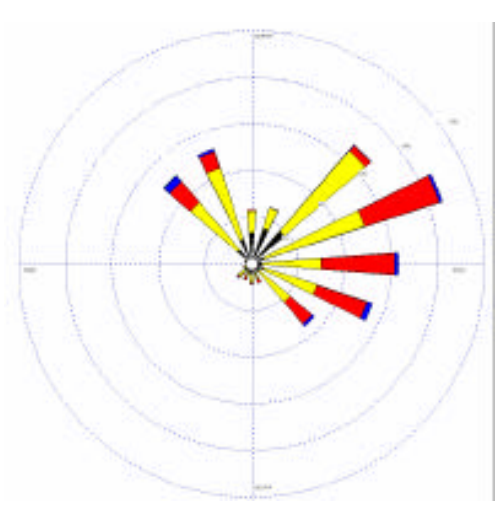

Dos Bocas

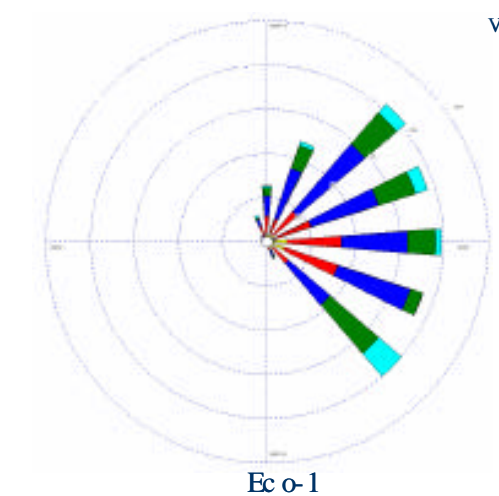

Ec $0-1$

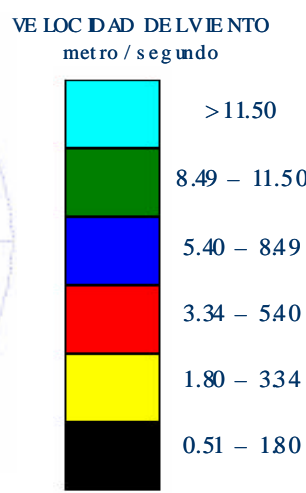

Figura 3. Rosas de viento para las estaciones meteorológicas D os bocas y ECO - 1 para el período de noviembre de 2000 a 2001

Para apreciar aún más la relevancia de la eficiencia de combustión en la estimación de las emisiones de quemadores elevados, se comparan los resultados presentados aquí con los obtenidos por Villasenor et al. (2003). La relación másica de $\mathrm{SO}_{2}$ a $\mathrm{H}_{2} \mathrm{~S}$ obtenida en nuestro estudio estuvo en el rango de 0.40 a 0.49 , mientras que el estudio que no factoriza el efecto de las eficiencias de combustión resulta en valores de 158 , es decir, se supone una eficiencia cercana al $100 \%$.

\section{Conclusiones}

En este trabajo se desarrolló un inventario de emisiones para quemadores elevados tipo antorcha operados por PEP en la RMNE. El inventario se construyó con base a información proporcionada por PEP y factores de emisión corregidos para to mar en cuenta la eficiencia de combustión de los quemadores, debido principalmente, a las condiciones meteorológicas locales. El inventario de emisiones se realizó para seis episodios a lo largo del período comprendido entre noviembre del 2000 y noviembre del 2001, con el fin de observar variaciones estacionales. En este aspecto, se observó una gran variabilidad en cuanto a las cantidades de emisiones atmosféricas de los quemadores de la RMNE, debido principalmente a que el volumen de gas quemado depende de aspectos operacionales durante la extracción y el transporte del gas natural, así como meteorológicos que impactan la eficiencia de los quemadores (principalmente velocidad del viento). Las eficiencias de combustión estimadas para los quemadores fueron bajas, por lo que se tienen emisiones considerables de $\mathrm{H}_{2} \mathrm{~S}$ y de hidrocarburos no quemados a la atmósfera. Estos resultados son consistentes, con lo que se puede esperar de manera teórica de la combustión de metano en quemadores tipo antorcha.

\section{Referencias}

Bravo H.A., Saavedra M.I.R., Sánchez P.A., Torres R.J. and Granada L.M.M. (2000). Chemical Composition of Precipitation in a Mexican Maya Region. Atmos. Environ., Vol. 34, N 0. 8, pp. 1197-1204.

Bravo H., Soto R., Sosa R., Sánchez P., Alarcón A.L. and Ruíz J. (2004). Effects of Wet Acidic Depo si tion on Stony Mate rial at the "Tajin" Archaeological Site in Veracruz, Mexico. In: Proceedings of the $97^{\text {th }}$ Annual Confer ence of the Air \& Waste Management Association, Indianapolis, IN, Paper N. 53.

ERG (Eastern Research Group, Inc.) (1999). M étodos para la estimación de emisiones al aire de campos de producción y operaciones de proceso de petróleo y gas, Volumen II, Capítulo 10.

Gamboa-Rodríguez M.T., Gamboa-Aldeco R., Abreu-Sherrer J.S., Bravo-Alvarez H. and Sosa-Echeverria R. (2004). Polynuclear Aromatic Hydrocarbons in the Air of an Oil Extrac tion Region in the South of México. In: Proceed ings of the $97^{\text {th }}$ Annual Conference of the Air \& Waste Management Association, Indi a nap olis, IN, Paper N 0. 470. 
matic Hydrocarbons in the Air of an Oil Extrac tion Region in the South of México. In: Proceed ings of the $97^{\text {th }}$ Annual Conference of the Air \& Waste Management Association, Indi a nap olis, IN, Paper No. 470.

Goodin W.R., McRae G.J. and Seinfeld J.H. (1979). A Comparison of Interpolation Methods for Sparse Data: Application to Wind and Concentration Fields. J. Appl. M eteor., Vol. 18, pp. 761-771.

Leahey D.M. and Schroeder M.B. (1987). Observations and Predictions of Jet Diffusion Flame Behavior. Atmos. Environ., Vol. 21, No. 4, pp. 777-784.

Leahey D.M., Preston K. and Strosher M. (2001). Theoretical and Observational Assessments of Flare Efficiencies. J. Air \& Waste Manage. Assoc., Vol. 51, No. 12, pp. 1610-1616.

Mendoza A. y Graniel M. (2004). Modelación del transporte de emisiones generadas en quemadores elevados de instalaciones petroleras en la Sonda de Campeche. En: Memorias del XXXIV Congreso de Investigación y Extensión del Tecnológico de Monterrey, Monterrey, México.

Parungo F., Nagamoto C., Hoyt S. and Bravo $\mathrm{H}$. (1990). The Investigation of Air Quality and
Acid Rain Over the Gulf of Mexico. Atmos. Environ., Vol. 24A, No. 1, pp. 109-123.

PEMEX (2004). Anuario estadístico 2003: PEMEX - Exploración y Producción. www.pep.pemex.com (revisado 23-mayo-05).

US EPA (United States Environ mentalProtection Agency) (1990). Health Assessment Document for Hydrogen Sulfide. EPA/ 600/8-86/026A, Office of Health and Environmental Assessment, Environmental Criteria and Assess ment Office. Research Triangle Park, NC.

US EPA (United States Environ mental Protection Agency) (1995). Compilation of Air Pollutant Emission Factors AP-42. $5^{\text {th }}$ Edition, Office of Air Quality Planning and Stan dards, Research Triangle Park, NC.

Villasenor R., Magdalena M., Quintanar A., Gallardo J.C., López M.T., Jurado R., Miranda A., Aguilar M., Melgarejo L.A., Palmerín E., Vallejo C.J. and Barchet W.R. (2003). An Air Quality Emisión Inventory of Offshore Oper a tions for the Exporation and Production of Petroleum by the Mexican Oil Industry. Atmos. Environ., Vol. 37, No. 26, pp. 3713-3729.

\section{Semblanza de los autores}

Alberto M endoza-Domínguez. Es profesor-investigador del Departamento de Ingeniería Química del ITESM Campus Monterrey y director del Centro de Tecnología Limpia del Centro de Calidad Ambiental del mismo Instituto. Obtuvo su grado de doctor en ingeniería ambiental del Georgia Insti tute of Tech nology. Sus áreas de investigación están relacionadas principalmente con modelación y monitoreo calidad del aire.

Manuel Graniel-Peralta. Obtuvo la licenciatura en ingeniería industrial en química en el Instituto Tecnológico de Mérida y el grado de maestro en ciencias del ITESM Campus Monterrey. Actualmente labora en la Gerencia de Seguridad Indus trial y Protección Ambiental de PEMEX Exploración y Producción, Región Marina Noreste. 